\title{
A Real-Time Interactive Shared System for Distance Learning
}

\author{
Xinyou Zhao Yanru Zhong \\ Mitsuji Matsumoto \\ Department of Computer Science Global Information and Telecommunication Studies \\ Guilin University of Electronic Technology \\ Waseda University \\ Guilin, China \\ Tokyo, Japan
}

Email: youzx@gliet.edu.cn

\begin{abstract}
The greatest obstacle in developing distance learning system is the lack of real-time interaction. This paper provides a real-time interactive shared system for distance learning that combines the audio, video and seminar. The remote learners can study and exchange opinions with the instructor through this system in real time. After the test between Waseda University and Guilin University, we find that the sistem can well simulate traditional classroom and improve interaction among participators, which not only saves learners' study time and improves their learning interests and efficiency, but also improves the teaching freedom of instructor for $e$-whiteboard is introducing.
\end{abstract}

Keywords: Distance Education, Distance Learning, Interaction, Network Education, E-learning

\section{Introduction}

Currently, because of constant changes in technology, continuous up-to-date education for IT workers in particular has become important ${ }^{[1][2][3]}$ However. these workers are often too busy to participate because their studying time coincides with working time. According to traditional educational patterns, these participators, including instructors and learners, don't have enough time staying at classroom because these courses require them to travel on campus and attend one class at specific times ${ }^{[5][7]}$.

When separated with instructor by space and/or time. learners staying far away can participate in a course on-line or off-line. This system is known as distance education system. Providing lifelong education through distance education system has become one of the most important roles in higher education.

With the rapid development of technology and current conditions, all educational organizations have been seeking a good way to offer more education opportunities without increasing educational costs. Distance education based on computer can make full use of the present educational manpower and material resources ${ }^{[2] ~[5] ~[9] ~[11] . ~ A ~ d i s t a n c e ~ e d u c a t i o n ~ s y s t e m ~}$ should offer a shared environment between participators. The environment should have two main characteristics $^{[7]}$ : (1) seeing \& hearing and (2) shared activity. Online education by using personal computers and networks is one of the most promising and attractive learning delivery methods today ${ }^{[2]}$. Presently distance education systems are mostly based on the Web to realize remote education ${ }^{[3][4][6]}$ ${ }^{[12]}$. But because these programs and courses are taught only in one direction, learners must passively allow themselves to be filled by knowledge and can't instantly discuss topics with the instructor [3] Although a few systems may already have got interaction between instructor and learners by adopting E-mail and BBS, these techniques can't solve the problem of face-to-face interaction.

In order to achieve face-to-face interaction for on-line teaching, video and audio communication between instructor and learners are needed. Baumgartner ${ }^{[3]}$ and Davis ${ }^{[6]}$ gave some systematic models based on audio and video. But at most time, the blackboard is much more important -- that is, data sharing is more important than communication of video and audio. An outstanding problem in distance education is the lack of real-time data interaction. Haga ${ }^{[1]}$ put forward one model that combined BBS with video so that participators could exchange ideas. Because audio is not embedded, this model can't well simulate traditional classroom to achieve the face-to-face study (question-answer) between instructor and learners. When participators want to 
offer relevant materials (such as pictures, documents, etc.) for discussion, Haga's model doesn't also work well.

This paper proposes a real-time interactive shared system for distance learning that combines the audio, video and seminar. Remote learners may instantly exchange opinions with the instructor through the system. Participators may exchange ideas through materials such as pictures and documents online. Moreover. the whiteboard is also introduced and improves the freedom of instructor for teaching. The system can well simulate traditional classroom.

The remainder of the paper is organized into four sections. Section 2 gives the system model of distance learning. Section 3 describes a real-time shared system. The performance of the system is analyzed by one experiment in section 4. The last section will look forward to the system.

\section{Distance Learning System Model}

Transmission technology plays a key role in the delivery of distance education, in which the course contents can be distributed to learners ${ }^{[2][8][10]}$. There are four main kinds of multimedia communication technology used in modern distance learning:

1) Audio Technology: Including the technology of speech, speech compression, etc.

2) Video Technology: Including the technology of image \& pictures, movie, animation, etc.

3) Data Transmission Technology: The computer sends and receives electronic messages such as courseware, E-mail, discussion, etc.

4) Print Transmission Technology: Printing is a basic element in distance learning. It provides a foundation for other teaching materials including textbooks, documents, etc.

These technologies can be divided into three general categories in distance education: data communication, video communication and audio communication. During learning, learners need audio and video to intercommunicate. But the data are as important as video \& audio because the data will always be accompanied with interpersonal communication. Only in few cases face-to-face intercourse is one-way between instructor and learners.

This paper proposes a model by combining video, data and audio, which can perform well under the management of control center. It may satisfy the learner's need. Learners can instantly communicate with instructor and peers. The model containing three exchanging space is shown in Fig. 2-1. They cooperatively work together under control center.

1) Data Space: In classroom, participators share information with a blackboard or pictures. In this model, they can exchange views by BBS discussion. electronic whiteboard, attached materials, etc.

2) Audio Space: Audio has played an important role in distance education for learners' questions and answers, instructor's lecture, etc.

3) Video Space: During study, classroom ambience is very important to learners, such as the instructor's gestures and expression, the responses of other learners, etc. .



Fig.2-1 System Model

\section{Real-Time Interactive Shared System}

The system consists of four parts. The first part is the control panel, by which learners can control the whole learning process, such as connecting, playing. etc. The second is a seminar where learners and instructor discuss questions and offer relevant materials. The third is the audio subsystem, and the fourth is the video subsystem. The system is shown in Fig. 3-1.

\subsection{Control Panel Subsystem}

The control panel (shown in Fig.3-1 (1)) contains connection control, playing control, sharing control, etc. Learners can connect to the instructor's server and may study on-line or off-line. When learners synchronistically study with instructor, the server will auto-send the courseware to them after they have successfully connected. While learners 


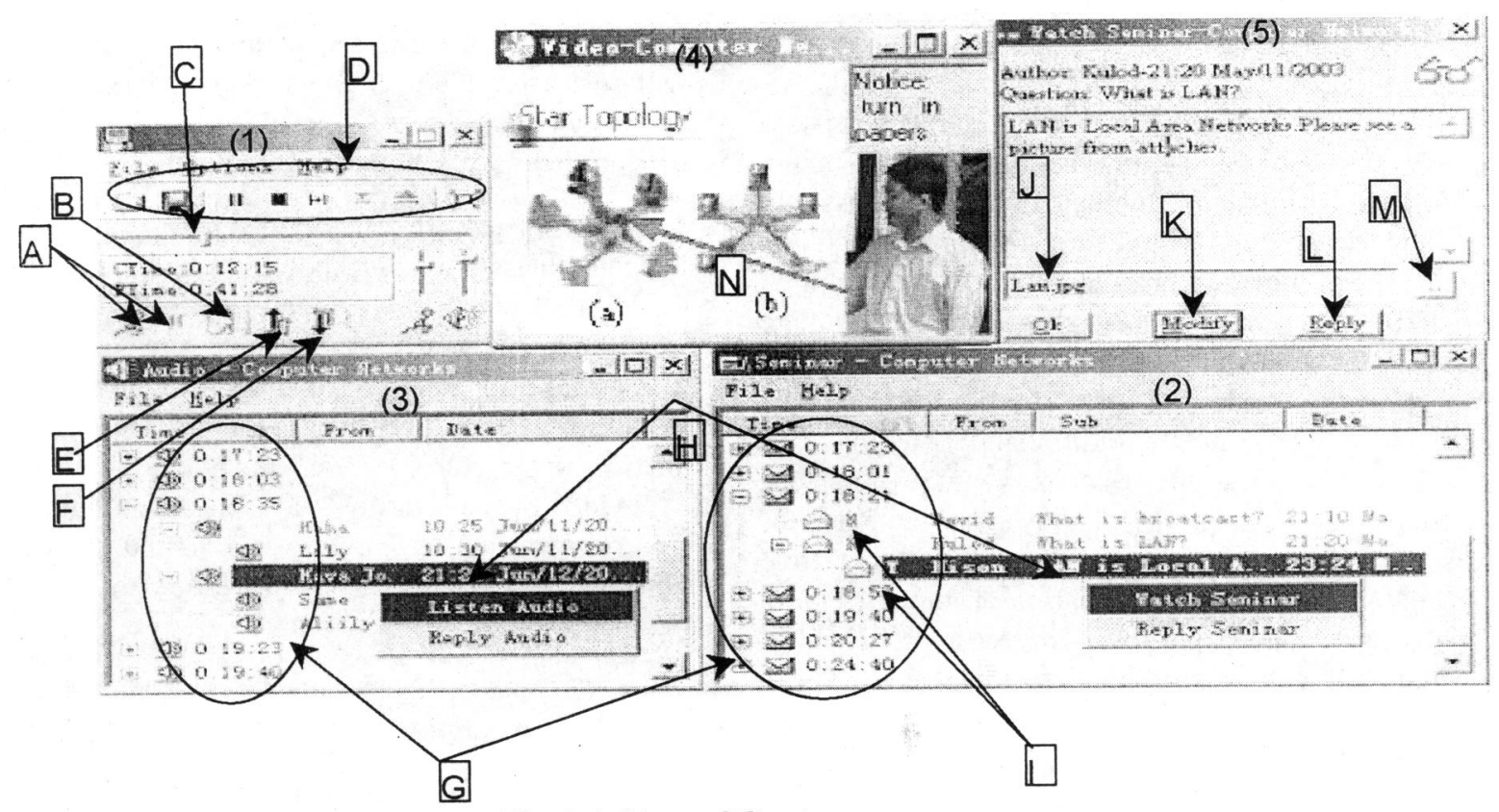

Fia.3-1 Shared System

asynchronously studying, courses will be played by stream media technology.

During studying off-line, the learner may use the time slider (Button $\mathrm{d}$ in Fig.3-1) to choose contents that he or she is interested in. Moreover, he or she may repeatedly learn selected parts, pause, save and watch in full screen mode by using some buttons (Button Group D in Fig.3-1). Sometimes, learners can download the materials to his computer from the server because of the networks' performance. While studying off-line, the learner may participate in a local discussion, and then he may upload (Button $\mathrm{E}$ in Fig. 3-1) his own opinion (including speech, seminar discussion and materials) to the instructor's server. When some other materials have been updated, he may download (Button $\mathrm{F}$ in Fig.3-1) new materials for studying.

\subsection{Seminar Subsystem}

As Fig. 3-1 (2) shows, participators can exchange their opinions \& ideas, which is similar to the BBS function. It has four components: time of corresponding video, author, subject and date. In order to show the relation between the discussion and the video being played, the time ( $\mathrm{G}$ in Fig.3-1) indicates the playing time of current video (such as corresponding discussions of video at 0:18:21).

If one meets a problem during study, he may sponsor a new question (Button B in Fig.3-1, the red indicates a new question, the blue indicates an answer) or look at other learners' opinions corresponding to the time of video. All questions and answers are sorted by time of video. He may also participate in other discussions ( $\mathrm{H}$ in Fig. 3-1).

When a question or answer can't be described clearly by texts, general BBS system can't do well. The system adopts a new way, which embeds files or pictures in the seminar (Button $M$ in Fig.3-1 uploads materials while discussing and saves materials while watching). There is an N/Y (I in Fig. 3-1) with every question time point, which shows whether there are materials for discussion. Thus, every learner can understand questions or answers easily.

The learner can go over all messages from seminar, participate in discussion (Button 凹 in Fig.3-1), edit or delete his message (Button $\mathbb{K}$ in Fig.3-1). If there are materials, the edit box (J) in Fig. 3-1) displays their names.

\subsection{Audio Subsystem}

As Fig.3-1 (3) shows, speech is embedded for discussion among learners and instructor. It contains three components: time of corresponding video, author and date. The time is similar to time of the seminar subsystem (such as discussions of video at $0: 18: 35$ ). The seminar subsystem manages text 
messages, and the audio deals with speech messages.

When the learner meets problems with the learning contents at any time, he or she can speak about questions (Button Group A in Fig.3-1), which are sorted by the time of course. Other learners can find questions by the time point (for example, video at $0: 18: 35$ has five audio materials, among which there are two new problems and three answers). The method has greatly facilitated discussion among learners. It saves inputting time of messages and improves learning efficiency. After learners create one virtual group while studying live, he may double-click the audio and also use right-click menus ( $\mathrm{H}$ in Fig.3-1) to listen or talk. Other learners, who have been authorized, can join the small group and freely participate in discussion. Of course, he must discard voice of instructor. These simulate the little quiet talk between classmates in traditional class.

When the instructor agrees with discussion at classroom, the system adopts the microphone seizing system to get question-answer interaction. The model is illustrated in Fig. 3-2. Four learners have sent requests to use audio resources at the same time, but the instructor can only respond to one of the requests and must ignore others. After the learner has monopolized the audio resources, he may discuss with the instructor. Others can only listen without speaking. This model is similar to traditional class. When the instructor provides one question and learners will voluntarily raise their hands, the instructor randomly chooses one of the learners to answer
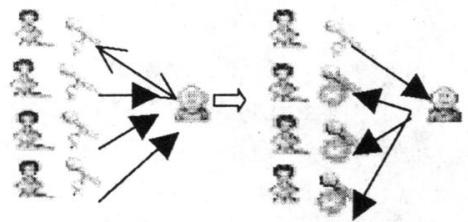

Fig.3-2 Microphone Seizing System

\subsection{Video Subsystem}

Currently instructor usually releases his course through one projector. The courseware may be designed in advance and can be distributed to the learners before the class begins. During class, the course video need not be transmitted to the learners' terminal. On the contrary, the real-time information about the instructor, such as facial expression, speaking mood, must be transmitted.
If the instructor's information is combined with the course contents, a high data rate is needed. For good efficiency of transmission, the system's video is split into two parts: course video and instructor video. which are shown in Fig.3-1 (4).

The video window contains three parts: notice board, course video and instructor video

1) Notice Board: The instructor \& learners may publish the news about the course, such as new contents, experimentation contents, test date etc.

2) Instructor Video: It is also called the instructor activity area at learner's terminal. In fact, there are two areas for instructor in this system, which are left area and right area. When the instructor is standing at the left of projector, his live video will be displaved at left area of learning system and the right area will be auto-hided (Shown at Fig.3-1 (4)), and vice versa.

Because the instructor's video is dynamic, it must instantly be updated. Another, the facial expression and gestures of instructor are much more important than others, such as foot's move. So one upper half and hands of instructor are only captured by camera. It will reduce the size of video data and improve the transmitting performance of distance learning system.

3) Course Video: At most times the course video is static during teaching. When the instructor changes contents of the course, the server will send one changing instruction to learners. After the learning system receives instructions, it will update the course video in accompany with the instructor.

In this system, instructor uses one touch screen. While the instructor is giving his course, he may point his key knowledge by his hands or one device. After the touch screen recognizes the touching point, server system will produce coordinates of touching point and transmit the coordinates to learners. After the learning system receives the coordinates, it will auto-produce one baton pointing the key point according to the size of learners video window $(\mathbb{N}$ in Fig.3-1). Another, when the instructor may use the electronic board (Instructor writes on the touch screen), the system only transmits coordinates of touching trace by the instructor. Then the learning system will re-draw the trace of instructor on the learner's terminal. It also reduces the need of network bandwidth for only coordinates of some points are transmitted. 


\section{Experiments on Shared System}

In order to evaluate the shared system, we did one test between Waseda University and Guilin University of Institute Technology on June 2005. The instructor gave his course at Waseda University and 19) volunteered learners studied at Guilin University (9 learners) and Waseda Honjo Campus (10 learners). The learners all gave much interest in the system. After we completed our experiment, we summarized the following benefits from participators.

1) Text Discussion: In traditional classroom, the learner can't discuss questions with classmates while they are listening because the discussion would produce noise, which disturbs other classmates. In the system, the learner can chat with other peers through seminar while studying (Button B in Fig.3-1). It will enable learners to actively participate in a virtual classroom rather than to passively listen to one lecture in a traditional virtual classroom. Moreover, they will be encouraged to deeply discuss topics of mutual interests, which can't happen in a traditional class. While studying on-line or off-line, they can share their views with other participators. These improve learners' understanding capability and lcarning efficiency.

2) Virtual Group Speech Discussion: When the instructor needs learners to take part in one discussion, he will release the audio resource. The instructor chooses one from all voluntary learners. The learner begins to monopolize the audio resources (Button A in Fig.3-1). This method simulates traditional class with face-to-face study. Moreover, learners can create one virtual group, whose members can only hear their group discussion. It is much better than simple one-way communication. It greatly improves interaction between learners and instructor.

3) E-learning Tools: The system is also one c-learning system because the server has recorded all information during class. Those learners who are not available at the class time may study at night or other times. If he finds himself getting distracted and thinking about other things, pull his attention back into a study groove as soon as possible. It will save time and improve learning efficiency.

4) Resource Self-Positioning: Whenever a learner watches the course video, it will automatically locate the audio and seminar resources because every discussion is corresponding to time of the lecture ((2) and (3) in Fig.3-1 $)^{[13]}$. Moreover, he may find corresponding messages and audio by the time $(\mathrm{G}$ in Fig.3-1). This greatly facilitates learners to learn and reduces his searching time.

5) Multi-Resource for Studying: If some questions cannot be clearly explained by texts, general BBS will not work well. In system, participators can upload materials (such as video, audio, documents. etc.) and help learners to understand.

6) Transmitting Performance: It adopts separation of instructor and course video. To instructor's video, only one part is transmitted. To course's video, it will be distributed in advance and changed by the instruction. When the instructor uses the whiteboard, only a series of coordinates of trace or point are sent. Based on these above, the system will not only need a low bit rate, but also improve real-time performance of interaction.

7) Learners Interests: The system can be used to judge learner's main interests ${ }^{[1]}$. The discussion density at some time of video indicates the quantity of comments around a specific topic. Therefore, learning interests can be easily determined. The real-time feedback enables instructors to make just-in-time decisions on instructional content to better meet learners' needs.

\section{Conclusion}

This paper proposes a real-time interactive shared system for distance learning by combining audio, video and seminar. The system improves real-time interaction between learners and instructor and provides a more personalized way of teaching and learning. But after test, the participators provided many good suggestions; for example, learners can't use e-board and participators can't see the response of other learners in real time, etc. In future, we will develop one embedding technology to realize one virtual classroom, which makes learners \& instructor not feel isolated again.

\section{Acknowledgments}

We would like to thank for their useful suggestions and comments to Kamugisha Kazaura and Professor G. E. Lindamood. 


\section{References}

[1] Hirohide Haga. Combining video and bulletin board system in distance education systems. Internet and Higher Education.2002, 5:119-129.

[2] A. Asif. Multimedia and cooperative learning in signal processing techniques in communications. Signal Processing Letters, IEEE. 2004(2), 11(2): 278-281.

[3] P. Baumgartner, S. Payr. Educating the knowledge worker in the information: basis support for efficient research. Teleteaching' 98 - Distance Learning, Training and Education -Proceedings part I.1998, 109-118.

[4] Felipe Baytelman, Nelson Baloian, Mitsuji Matsumoto. Remote-lecture cooperative software system for the computer-Integrated classroom. The IEEE Region

10 Conference on TENCON 2004(Thailand). 2004(11): 259-261.

[5] K.Chassie. The allure of $\mathrm{e}$ learning. IEEE Potentials.2002 (8), 21(3): 33-35.

[6] James L.Davis, Thomas W.Smith. Computer-assisted distance learning technique in Japanese instruction. 1992 Frontiers in Education Conference.1992: 683-687.

[7] Keegan D. The foundations of distance education. Croom Helm. 1986.
[8] David Jones. Computing by distance education: problem and solutions. ACM SIGCSE Bulletin.1996, 28:139-146.

[9] M. Nycz, B. Smok. Distance education as a way to meet the challenges of the XXI century. Mobile Future and Symposium on Trends in Communications. 2003(10): 163-166.

[10] Takao Ichiko, Masaki Yamamoto, Yousuke Kawamura, Motochika Hanano. Advanced multimedia telecommunications using a high-speed broadband backbone network beyond all aspects of the current Internet. Computer \& Education 2001,37:211-224.

[11] Lisa C. Kaczmarczyk. Accreditation and student assessment in distance education: why we all need to pay attention (poster session). ACM SIGCSE Bulletin.2001.33 (3): 113-116.

[12] June Lu, Chun-Sheng Yu, Chang Liu. Leaming style, learning patterns, and learning performance in a WebCT-based MIS course. Information and Management.2003 (7), 40(6): 497- 507.

[13] Finke, M., Wichert, R., Balfanz, D. An Interactive Video System for Live Coverage Transmission using Real Time Video Hyperlinks. Multimedia, Internet, Video Technologies 2001. 